\title{
Enfrentando el dilema de las suspensiones: características e incidencia de las suspensiones quirúrgicas en un centro académico en Chile
}

\author{
Juan Carlos Pattillo S. ${ }^{1}$ y Franklin Dexter ${ }^{2}$
}

'Sección Cirugía Pediátrica División de Cirugía, Facultad de Medicina, Pontificia Universidad Católica de Chile Santiago, Chile.

2Division of Management

Consulting, Department of Anesthesia, University of lowa, lowa City, lowa, USA.

Recibido el 24 de septiembre de 2017 y aceptado para publicación el 13 de diciembre de 2017

Correspondencia a: Dr. Juan Carlos Pattillo S. jpattill@med.puc.cl
Facing the dilemma of cancellations: incidence and characteristics of surgical cancellations in a Chilean academic hospital

Introduction: Surgical cancellations are a complex problem in the field of Operating Room Management, affecting patients, staff, including surgeons and anesthetists, and compromising the finances of health institutions. The available information about the incidence and characteristics of surgical cancellations in our country are scarce. Objective: The aim of this article is to characterize the surgical cancellations in a Chilean academic center, describing its global incidence and by specialty. Materials and Methods: Prospective information on surgical schedule and Operating Room activity was included in this cohort study of surgical case cancellations occurring from January 1, 2016 through May 20, 2017. To calculate the percentage of cancellations, 3 groups were used based on 6 periods of 4 weeks. The Freeman-Turkey double-arcsine transformation and Student's t-test were applied. Results: During the study period, 11,398 surgeries were programmed, of which 492 were cancelled. The incidence of cancellations for the 3 periods was $4.38 \%$ (95\% CI $3.78 \%$ to $5.01 \%), 4.15 \%(95 \%$ CI $3.51 \%$ to $4.85 \%)$ and $4.10 \%(95 \%$ CI of $3.50 \%$ to $4.74 \%$ ) respectively. In at least $57 \%$ of cases the main identifiable cause of cancellation was a change in the patient's medical condition. Discussion and Conclusions: Our results consistently show that the cancellation rates range between $3 \%$ and $5 \%$, which is within international standards, and can be used as benchmarking for comparisons at a national level.

Key words: elective surgery; surgery cancellation; operating room management.

\section{Resumen}

Introducción: Las suspensiones quirúrgicas constituyen una problemática compleja en el ámbito de la administración de pabellones, que afecta tanto a pacientes como al personal de pabellón, y que compromete la eficiencia financiera de las instituciones de salud. Existe escasa información acerca de la incidencia y características de las suspensiones quirúrgicas en nuestro país. Objetivo: Caracterizar las suspensiones quirúrgicas en un centro académico chileno, describiendo su incidencia global y por especialidad. Materiales y Métodos: Se incluyó información recopilada de manera prospectiva acerca de la programación y actividad de pabellón, y de una cohorte de pacientes suspendidos de cirugía entre el 1 de enero de 2016 y el 20 de mayo de 2017. Para calcular el porcentaje de suspensiones se utilizaron 3 grupos en base a 6 períodos de 4 semanas. Se aplicó la transformación de doble arcoseno de Freeman-Turkey y el test-t de Student. Resultados: Durante el período del estudio se programaron 11.398 cirugías, de ellas 492 fueron suspendidas. La incidencia de suspensiones de los 3 períodos fue de 4,38\% (IC 95\% de 3,78\% a 5,01\%), $4,15 \%$ (IC $95 \%$ de $3,51 \%$ a $4,85 \%$ ) y $4,10 \%$ (IC $95 \%$ de $3,50 \%$ a $4,74 \%$ ) respectivamente. Al menos en un 57\% de los casos la principal causa de suspensión se identificó como un cambio en la condición médica del paciente. Discusión y Conclusiones: Nuestros resultados muestran, de manera consistente, que las suspensiones se encuentran entre un $3 \%$ y un $5 \%$, lo que está dentro de los estándares internacionales, y pueden ser utilizados como un "benchmarking" para comparaciones a nivel nacional.

Palabras clave: cirugía electiva; suspensión de cirugía; administración de pabellones. 


\section{Introducción}

La suspensión de un caso electivo en el día de la cirugía es una situación que repercute en la satisfacción de los pacientes, en la eficiencia de los sistemas de salud y en la sociedad. Los costos, tanto para los equipos médicos, como para los pacientes y el sistema hospitalario, son mayores al tener que reprogramar un caso que al resolverlo, incluso cuando se produzca sobreutilización de recursos ${ }^{1,2}$.

La cancelación de una cirugía repercute también en los acompañantes de los pacientes, quienes incrementan su ausentismo laboral, siendo esta observación válida tanto para pacientes adultos como pediátricos ${ }^{3}$.

Al enfrentar un porcentaje elevado de suspensiones, los encargados de la tabla quirúrgica suelen resolver el problema citando a los pacientes más temprano el día de la cirugía, para poder llenar los espacios que eventualmente se produzcan durante el desarrollo de la tabla. Esta conducta aumenta los tiempos de espera y podría generar insatisfacción en los pacientes ${ }^{4-7}$.

Nuestro hospital es un centro académico de 470 camas. De estas, el $30 \%$ corresponde a camas de alta o mediana complejidad y el $20 \%$ corresponde a camas destinadas a pacientes pediátricos. Nuestra institución cuenta con 25 pabellones, incluyendo maternidad, en los que durante el año 2016 se realizaron 30.893 procedimientos. Entre estos, hubo 1.009 tipos distintos de procedimientos; $67,4 \%$ fueron realizados de manera frecuente (desviación estándar 1,0$)$, mientras que el $28,6 \%$ correspondió a procedimientos tan infrecuentes, que sólo se realizaron 1 o dos veces. El $67^{\circ}$ procedimiento más realizado correspondió a un $0,29 \%$ de los procedimientos realizados. De esta forma, podemos caracterizar a nuestro hospital como un centro académico-docente de gran tamaño y alta complejidad, en el que se realiza una gran diversidad de procedimientos.

El Servicio de Pabellón de Cirugía General cuenta con 13 pabellones y concentra a las especialidades de cirugía digestiva (CDG), traumatología y ortopedia (TMT), urología (URO), cirugía oncológica, de cabeza y cuello y máxilofacial (ONC), cirugía pediátrica (CPED), cirugía plástica (CPLAS), otorrinolaringología (ORL) y ginecología oncológica (GINE). Entre el $75 \%$ y el $80 \%$ de las cirugías realizadas en el Servicio corresponden a cirugías programadas. En este Servicio, el porcentaje de suspensiones estimado entre junio y noviembre de 2014 alcanzaba un $8,3 \%$ (Intervalo de confianza 95\% de $5,2 \%$ a $12,1 \%$ ) y entre abril y octubre de 2015 llegaban a un 6,9\% (Intervalo de confianza 95\% de
$4,6 \%$ a $9,7 \%$ ). Estas estimaciones fueron calculadas en estudios de consultoría previos realizados por uno de los autores (F.D. CalculatOR Report 2015 y 2016$)^{8,9}$.

Durante el año 2015, se implementó un sistema de programación centralizada de la tabla quirúrgica, planteándose como una de las metas el control de las suspensiones de cirugías electivas, en busca de una mayor satisfacción de nuestros pacientes y equipos médicos. Este artículo tiene como objetivo estimar la incidencia y caracterizar las suspensiones quirúrgicas en un centro académico chileno.

\section{Materiales y Métodos}

El estudio propuesto corresponde a un estudio descriptivo de una cohorte. Fue presentado al Comité de Ética y Seguridad en Investigación obteniéndose una dispensa al no involucrar personas, datos personales y/o sensibles, ni participación de seres vivos o uso de materiales, tangibles o intangibles, especialmente protegidos en la investigación científica (ID proyecto 170517003).

Durante el año 2015, en el Servicio de Pabellón de Cirugía General, comenzó la implementación de un sistema de programación centralizada de la tabla quirúrgica, independiente de los departamentos académicos como era hasta ese momento. Básicamente se trata de un sistema de "first-call first-served", es decir, se prioriza por orden de llegada, pero se mantiene la asignación estratégica de bloques por cirujano o servicios quirúrgicos, para facilitar la programación y el uso más eficiente del pabellón.

Las principales características de esta programación son:

1) La asignación del tiempo de pabellón lo realiza la enfermera programadora en base a la combinación "cirujano-procedimiento"

2) La enfermera programadora asigna el pabellón por orden de llegada.

3) Los equipos quirúrgicos tienen bloques asignados que aseguran el acceso a primera hora a cambio de una menor flexibilidad en la programación. Existe el compromiso de llenar primero los bloques y de acceder a otros espacios en la programación, una vez que esto ha ocurrido.

4) Los bloques se liberan $48 \mathrm{~h}$ antes de la cirugía, momento en que la tabla queda abierta para programar casos, siempre por orden de llegada. La administración de pabellón deja un pabellón subutilizado de manera consistente para poder resolver las urgencias o pacientes que se agreguen a la tabla. 


\section{ARTÍCULO ORIGINAL}

Cuando se recibe una solicitud de pabellón, en la oficina de programación se gatilla un proceso que incluye la asignación de una fecha probable de pabellón, se refuerza la solicitud de donantes de sangre de acuerdo a las políticas internas de reserva de hemoderivados según el tipo de cirugía, y se comienza con la educación del paciente acerca del proceso perioperatorio. Simultáneamente, se coordina la entrega de insumos y materiales específicos para cada cirugía. Dependiendo de la cercanía a la fecha de cirugía y previo a la publicación de la tabla quirúrgica, se confirma la condición médica del paciente, se refuerzan las instrucciones perioperatorias y se completa la educación del paciente. Al mediodía del día hábil previo al día de la cirugía se publica la tabla quirúrgica.

Durante el día de la cirugía el manejo de la tabla quirúrgica lo realiza la enfermera coordinadora del pabellón, dando espacio a los pacientes agregados de acuerdo a las prioridades que permiten la mayor eficiencia en el uso del tiempo de pabellón ${ }^{12}$.
El servicio de pabellón cuenta con un sistema informático basado en Filemaker Pro 6.0 (Copyright C 1994-2017, FileMaker, Inc, FileMaker International). Cuando ocurre una suspensión se llena un formulario electrónico en el que se especifica la causa de ésta ${ }^{13}$. Semanalmente se recopila la información de los pacientes que han sido programados, los pacientes que han sido suspendidos y las causas de suspensión, generando un informe que se distribuye a los cirujanos y administradores relacionados con el servicio. Se considera como suspendido un paciente que, estando programado con al menos $24 \mathrm{~h}$ de anticipación, no se opera en el día de la cirugía. De esta definición se excluyen los cambios en la programación que ocurren antes de la publicación de la tabla-los que no tienen un impacto relevante en la eficiencia en el uso de pabellones-, así como los cambios que ocurran en pacientes que son agregados a la tabla una vez que esta ha sido publicada ${ }^{14}$. Respecto de los motivos de suspensión, estos fueron clasificados como se muestra en la Tabla 1, modificada de Argo 2009.

Tabla 1. Clasificación de las causas de suspensión de cirugía. Modificado de Argo 2009

\begin{tabular}{|c|c|c|}
\hline \multirow[t]{2}{*}{ Paciente } & $\mathrm{P} 1$ & Paciente se niega o no da consentimiento \\
\hline & $\begin{array}{l}\text { P2 } \\
\text { P3 } \\
\text { P4 } \\
\text { P5 } \\
\text { P6 } \\
\text { P7 } \\
\text { P8 } \\
\text { P9 } \\
\text { P10 }\end{array}$ & $\begin{array}{l}\text { Transporte interno no disponible } \\
\text { Transporte del paciente no disponible } \\
\text { Paciente no sigue instrucciones preoperatorias o no ha sido instruido adecuadamente } \\
\text { Paciente con consumo de medicamento } \\
\text { Paciente suspende, procedimiento realizado en otra institución } \\
\text { Paciente suspende, procedimiento no realizado en otra institución } \\
\text { Paciente fallecido } \\
\text { Cirugía suspendida en pabellón } \\
\text { Paciente no se presenta, paciente no ubicable }\end{array}$ \\
\hline Enfermedad (Condición médica) & $\begin{array}{l}\text { E1 } \\
\text { E2 } \\
\text { E3 } \\
\text { E4 } \\
\text { E5 }\end{array}$ & $\begin{array}{l}\text { Evaluación de cirujano requerida } \\
\text { Evaluación de anestesia requerida } \\
\text { Exámenes alterados } \\
\text { Cambios en la condición médica } \\
\text { Cambio en el plan de tratamiento médico }\end{array}$ \\
\hline Institución & $\begin{array}{l}\text { I1 } \\
\text { I } \\
\text { I3 } \\
\text { I4 } \\
\text { I5 } \\
\text { I6 } \\
\text { I7 } \\
\text { I8 } \\
\text { I9 } \\
\text { I10 } \\
\text { I1 } \\
\text { I1 }\end{array}$ & $\begin{array}{l}\text { Equipo médico no disponible / roto } \\
\text { Implante (s) no disponibles } \\
\text { Falta de disponibilidad de camas UCI / Intermedio } \\
\text { Falta de disponibilidad de camas hospital } \\
\text { Error de programación } \\
\text { Falta de personal de pabellón (No se considera cirujanos ni profesional del área de anestesia) } \\
\text { Falta de disponibilidad de pabellón } \\
\text { Casos urgentes } \\
\text { Productos sanguíneos no disponibles } \\
\text { Problema con instalaciones } \\
\text { Desastres naturales o climáticos } \\
\text { Traslado a otro pabellón }\end{array}$ \\
\hline Anestesia & A1 & Anestesista no disponible \\
\hline Cirujano & $\mathrm{C} 1$ & Cirujano no disponible \\
\hline
\end{tabular}


ARTíCULO ORIGINAL

Es relevante hacer notar que esta clasificación es una sobresimplificación del problema de las suspensiones, y solo permite estimar la causa principal o el motivo al que se atribuye la suspensión al momento de realizarla, ya que muchas veces estos motivos se pueden sobreponer ${ }^{7}$. Por ejemplo, en el caso de un paciente alérgico al látex que se suspende, cuya alergia no fue informada, se podría atribuir a un error de programación, a una falta de disponibilidad de pabellón (que no fue debidamente preparado), a la necesidad de evaluación anestésica o a la falta de disponibilidad del cirujano que no quiso esperar que el pabellón se preparara, en caso de ser posible; en el caso de un cambio en la condición médica, por ejemplo, en un paciente con deterioro de su condición y que requiera cama UCI posoperatoria, la suspensión podría atribuirse a la falta de cama UCI o al cambio en la condición clínica, siendo ambos válidos. De esta forma, la clasificación nos permite entender el mínimo de suspensiones atribuibles a una causa.

Con la información disponible, calculamos el porcentaje total de suspensiones, las suspensiones por especialidad, así como sus intervalos de confianza y sus causas. Para este estudio se incluyeron todos los pacientes programados en el Servicio de Pabellón de Cirugía General entre el 1 de enero de 2016 y el 20 de mayo de 2017. Para calcular el porcentaje de suspensiones se utilizaron 3 grupos en base a 6 períodos de 4 semanas, realizando la transformación de doble arcoseno de Freeman-Turkey y aplicando el test $t$ de Student ${ }^{8,9}$. Se utilizó la definición de suspensión quirúrgica descrita más arriba, excluyéndose aquellos pacientes que corresponden a cambios de la programación y que no tienen un impacto operacional en el manejo diario del pabellón.

\section{Resultados}

Durante el período del estudio se programaron 11.398 pacientes, de ellos 492 fueron suspendidos. Para la estimación del porcentaje de suspensiones, se analizaron las suspensiones en 3 grupos de 6 períodos de 4 semanas, cuyas estimaciones puntuales e intervalos de confianza se encuentran en la Tabla $2^{8,9}$. Observamos que para todo el período del estudio, el percentil 95 de las suspensiones se encuentra entre un $3,50 \%$ y $5,01 \%$.

En la Tabla 3 se muestran las estimaciones puntuales e intervalos de confianza para las suspensiones por especialidad, incluyendo todas las suspensiones del período de estudio.

La Figura 1 muestra la causa principal de sus-
Tabla 2. Resultados de estimación puntual del porcentaje de suspensiones e intervalos de confianza del $95 \%$ para cada uno de los 3 grupos de 6 períodos de 4 semanas

\begin{tabular}{|lcc|}
\hline Período & \% Suspensiones & Intervalo de confianza 95\% \\
\hline $4 / 1$ al $19 / 6$ de 2016 & 4,38 & $3,78-5,01$ \\
\hline 20/6 al 4/12 de 2016 & 4,15 & $3,51-4,85$ \\
\hline $5 / 12$ de 2016 al $20 / 5$ de 2017 & 4,10 & $3,50-4,74$ \\
\hline
\end{tabular}

Tabla 3. Resultados de estimación puntual del porcentaje de suspensiones e intervalos de confianza del $95 \%$ para cada especialidad

\begin{tabular}{|ccc|}
\hline Especialidad & \% Suspensiones & Intervalo de confianza 95\% \\
CDG & 3,28 & $2,39-4,29$ \\
CPED & 5,45 & $3,53-7,73$ \\
\hline CPLAS & 5,32 & $3,10-8,02$ \\
GINE & $3,47^{*}$ & - \\
\hline ONC & 2,73 & $1,93-3,66$ \\
ORL & 5,12 & $3,56-6,92$ \\
TMT & 5,62 & $4,30-7,09$ \\
URO & 4,01 & $2,87-5,31$ \\
\hline
\end{tabular}

*Para la especialidad de GINE se muestra sólo el porcentaje de suspensiones crudo. No es posible estimar intervalos de confianza ya que hubo al menos un período de 6 semanas con menos de 2 suspensiones.

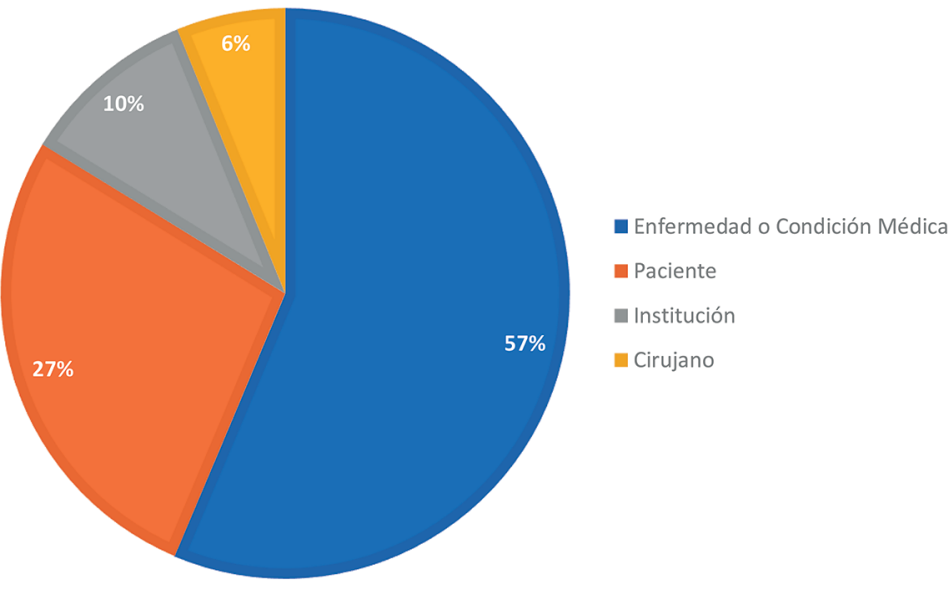

Figura 1. Causa Principal de suspensión en 492 cirugías. Se muestra la distribución de las cirugías suspendidas de acuerdo a la causa primaria de la suspensión. Ver discusión en el manuscrito. 


\section{ARTÍ́CULO ORIGINAL}

pensión de acuerdo a lo indicado al momento de suspender la cirugía.

Al menos en 276 casos (57\%) la suspensión de la cirugía se debió a un cambio en la condición médica o en el plan de manejo del paciente. Al menos 133 suspensiones (27\%) fueron generadas por los pacientes, ya sea porque realizaron un cambio de fecha $(n=74,15 \%)$ o de prestador $(n=23,4,6 \%)$; estas situaciones se debieron, en la mayoría de los casos, a problemas de cobertura previsional no resueltos de manera oportuna. Hubo por lo menos 17 pacientes $(3,4 \%)$ que no siguieron las instrucciones preoperatorias de manera adecuada y debieron ser suspendidas.

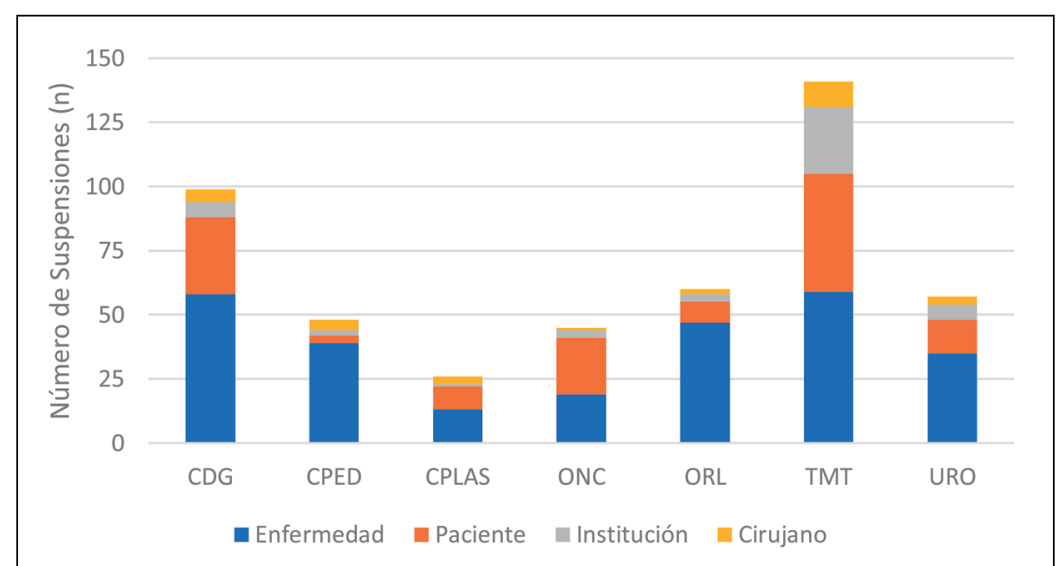

Figura 2. Causa de suspensión por especialidad. Se muestra el número absoluto de suspensiones por especialidad. La especialidad de GINE se ha excluido por tener un número muy bajo de suspensiones.

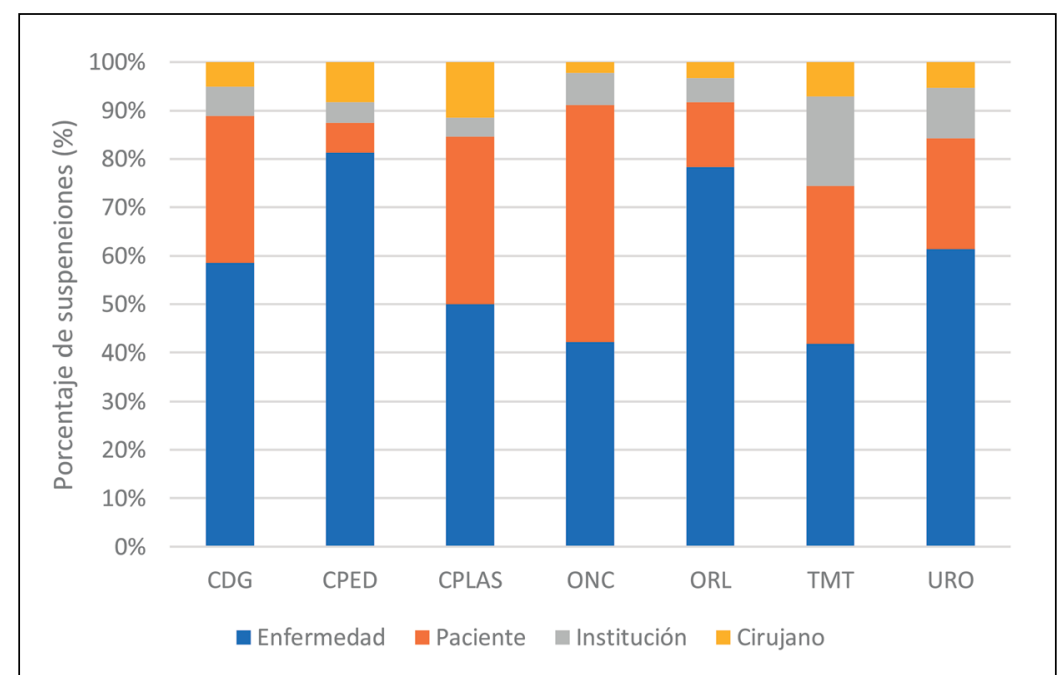

Figura 3. Distribución porcentual de las causas de suspensión por especialidad. Se muestra la distribución porcentual de suspensiones por especialidad. La especialidad de GINE se ha excluido por tener un número muy bajo de suspensiones.
Las suspensiones debido a falencias institucionales fueron por lo menos $50(10 \%)$. De ellas, 19 $(3,9 \%)$ se debieron a falta de disponibilidad de implantes o de instrumental específico, el mismo número a falta de disponibilidad de camas de hospitalización y $10(2 \%)$ a errores de programación.

Hubo no menos de 30 pacientes suspendidos por falta de disponibilidad del cirujano y no hubo suspensiones por falta de anestesistas.

En las Figuras 2 y 3 se muestran las suspensiones por especialidad, de acuerdo a la causa principal de suspensión. La Figura 2 muestra las suspensiones en números absolutos y en la Figura 3 se muestra de manera porcentual.

\section{Discusión}

En primer lugar, quisiéramos destacar que la literatura científica acerca de las suspensiones de cirugías en nuestro país es escasa ${ }^{15}$. Así mismo, el tema de la oportunidad de acceso a la atención médica, las listas de espera por cirugías y el uso eficiente de recursos de los servicios de salud, aparecen de manera recurrente en la prensa y en el debate público nacional, por lo que nos ha parecido relevante contribuir a entender la realidad de las suspensiones quirúrgicas desde el punto de vista científico, en un centro académico, que se ha puesto como objetivos optimizar el uso de los recursos.

El problema de las suspensiones comienza por entender su definición: una cirugía suspendida es aquella que, estando programada -al menos desde el día previo- no se realiza el día en que estaba planificado hacerla ${ }^{12,16}$. Esta definición es relevante pues lleva implícito el uso eficiente de recursos, por eso se excluyen los cambios de la tabla quirúrgica que ocurren antes de su publicación, así como los pacientes agregados a la tabla, que deben ser resueltos a la brevedad ${ }^{17}$.

Nuestros resultados muestran de manera consistente que las suspensiones se encuentran entre un $3 \%$ y un $5 \%$, lo que está dentro de los estándares internacionales y puede ser utilizado como un "benchmarking", para comparaciones a nivel nacional ${ }^{18,19}$

En cuanto a los motivos de suspensión, sabemos que existe una limitación en el análisis debido a la superposición de causas ${ }^{7}$. Sin embargo, es interesante hacer notar que existe una oportunidad para disminuir porcentaje de suspensiones en los casos gatillados por los pacientes. Esto debido a que, entre ellas, un número importante se debió a falencias en los procesos administrativos, como la solicitud y 
entrega de presupuestos, la realización de documentación para el ingreso administrativo o el desconocimiento de la cobertura por parte de los seguros de salud del paciente ${ }^{20}$. También podemos apreciar que, en números absolutos (Figura 2), los pacientes de los traumatólogos tienen un gran número de suspensiones por motivos "institucionales" (como falta de implantes o de equipos), y que los grupos que atienden pacientes pediátricos de manera frecuente (cirugía pediátrica y otorrinolaringología), tienen mayor número relativo de suspensiones debido a cambios en la condición médica, probablemente, debido a enfermedades respiratorias intercurrentes (Figura 3).

Finalmente, creemos que es importante que las autoridades de salud y los administradores de servicios quirúrgicos apliquen metodologías apropiadas para la estimación y monitorización de las suspensiones quirúrgicas ${ }^{8}$. También es necesario que se establezcan metas de acuerdo a estándares objetivos, y que para ello el proceso de programación y uso eficiente de pabellones se base en las mejores prácticas disponibles.

\section{Responsabilidades éticas}

Protección de personas y animales. Los autores declaran que para esta investigación no se han realizado experimentos en seres humanos ni en animales.

Confidencialidad de los datos. Los autores declaran que han seguido los protocolos de su centro de trabajo sobre la publicación de datos de pacientes.

Derecho a la privacidad y consentimiento informado. Los autores declaran que en este artículo no aparecen datos de pacientes.

\section{Conflicto de intereses}

JCP es empleado por la Facultad de Medicina de la Pontificia Universidad Católica de Chile y por la Red de Salud UC-CHRISTUS. Los cálculos descritos en este artículo fueron realizados por FD, perteneciente a The Division of Management Consulting, Department of Anesthesia, University of Iowa, utilizando métodos previamente publicados. FD no recibe otros pagos personales más que su sueldo y el reembolso de gastos por parte de la Universidad de Iowa, y tienen un contrato sin programa de incentivos. Él y su familia no tienen relación financiera con ninguna compañía relacionada con su trabajo, excepto indirectamente a través de fondos mutuos para la jubilación. Los ingresos generados para la División por consultorías son usados para el financiamiento de investigaciones científicas.

\section{Financiación}

No hubo financiamiento de fuentes concursables para este artículo.

\section{Bibliografía}

1. Tessler MJ, Mitmaker L, Wahba RM, Covert CR. Patient flow in the postanesthesia care unit: an observational study. Can J Anaesth. 1999;46:348-51.

2. Stepaniak PS, Mannaerts GH, de Quelerij $\mathrm{M}$, de Vries $\mathrm{G}$. The effect of the operating room coordinator's risk appreciation on operating room efficiency. Anesth Analg. 2009;108: 1249-56

3. Tait AR, Voepel-Lewis T, Munro HM, Gutstein HB, Reynolds PI. Cancellation of pediatric outpatient surgery: economic and emotional implications for patients and their families. J Clin Anesth. 1997;9:2139.

4. Wachtel RE, Dexter F. A simple method for deciding when patients should be ready on the day of surgery without procedure-specific data. Anesth Analg. 2007;105:127-40.

5. Smallman B, Dexter F. Optimizing the arrival, waiting, and NPO times of children on the day of pediatric endoscopy procedures. Anesth Analg. 2010;110:87987.

6. Dexter F, Traub RD. Statistical method for predicting when patients should be ready on the day of surgery. Anesthesiology 2000;93:1107-14.

7. Tung A, Dexter F, Jakubczyk S, Glick DB. The limited value of sequencing cases based on their probability of cancellation. Anesth Analg. 2010;111:749-56.

8. Dexter F, Marcon E, Epstein RH, Ledolter J. Validation of statistical methods to compare cancellation rates on the day of surgery. Anesth Analg. 2005;101:465-73 and erratum 2012;114:693.
9. Miller JJ. The inverse of the FreemanTukey double arcsine transformation. Am Stat. 1978;32:138.

10. Dexter F, Ledolter J. Bayesian prediction bounds and comparisons of operating room times even for procedures with few or no historical data. Anesthesiology 2005; 103:1259-67.

11. Dexter F, Epstein RH, Bayman EO, Ledolter J. Estimating surgical case durations and making comparisons among facilities: identifying facilities with lower anesthesia professional fees. Anesth Analg. 2013;116:1103-1115.10

12. Dexter F, Epstein RH, Traub RD, Xiao Y. Making management decisions on the day of surgery based on operating room efficiency and patient waiting times. Anesthesiology 2004;101:1444-53.

13. Argo JL, Vick CC, Graham LA, Itani 


\section{ARTÍ́CULO ORIGINAL}

KM, Bishop MJ, Hawn MT. Elective surgical case cancellation in the Veterans Health Administration system: identifying areas for improvement. Am J Surg. 2009;198:600-6.

14. Epstein RH, Dexter F. Management implications for the Perioperative Surgical Home related to inpatient case cancellations and add-on case scheduling on the day of surgery. Anesth Analg. 2015;121:206-18.

15. Promis DG. Gestión de un servicio quirúrgico. Rev Chil Cir. 1994;46:463-9.

16. McIntosh C, Dexter F, Epstein RH. The impact of service-specific staffing, case scheduling, turnovers, and first-case starts on anesthesia group and operating room productivity: a tutorial using data from an Australian hospital. Anesth Analg. 2006;103:1499-516.

17. Shi P, Dexter F, Epstein RH. Comparing policies for case scheduling within one day of surgery by Markov chain models. Anesth Analg. 2016;122:526-38.

18. Schuster M, Neumann C, Neumann K, Braun J, Geldner G, Martin J, et al. The effect of hospital size and surgical service on case cancellation in elective surgery: results from a prospective multicenter study. Anesth Analg. 2011;113:578-85.

19. Yu K, Xie X, Luo L, Gong R. Contributing factors of elective surgical case cancellation: a retrospective crosssectional study at a single-site hospital. BMC Surg. 2017;11;17:100.

20. Morris AJ, McAvoy J, Dweik D, Ferrigno M, Macario A, Haisjackl M. Cancellation of elective cases in a recently opened, tertiary/quaternary-level hospital in the Middle East. Anesth Analg. 2017;125:26877. 